\title{
SENSITIVITY ANALYSIS FOR SYSTEM OF PARAMETRIC GENERALIZED QUASI-VARIATIONAL INCLUSIONS INVOLVING $R$-ACCRETIVE MAPPINGS
}

\author{
Kaleem Raza Kazmi, Faizan Ahmad Khan†, and Naeem Ahmad
}

\begin{abstract}
In this paper, using proximal-point mappings technique of $R$ accretive mappings and the property of the fixed point set of set-valued contractive mappings, we study the behavior and sensitivity analysis of the solution set of the system of parametric generalized quasi-variational inclusions involving $R$-accretive mappings in real uniformly smooth $\mathrm{Ba}$ nach space. Further under suitable conditions, we discuss the Lipschitz continuity of the solution set with respect to parameters. The technique and results presented in this paper can be viewed as extension of the techniques and corresponding results given in $[3,23,24,32,33,34]$.
\end{abstract}

\section{Introduction}

Variational inequality theory introduced by Stampacchia [35] and Fichera [17] independently, in the early sixties in potential theory and mechanics, respectively, constitutes a significant extension of variational principles. It has been shown that the variational inequality theory provides the natural, descent, unified and efficient framework for a general treatment of a wide class of unrelated linear and nonlinear problems arising in elasticity, economics, transportation, optimization, control theory and engineering sciences $[5,6,10,18-20]$.

In 1968, Brezis [7] initiated the study of the existence theory of a class of variational inequalities later known as variational inclusions, using proximal point mappings due to Moreau [27]. Variational inclusions include variational, quasi-variational, variational-like inequalities as special cases. For application of variational inclusions, see for example $[4,13]$.

Received May 9, 2008.

2000 Mathematics Subject Classification. 49J40, 65K10.

Key words and phrases. system of parametric generalized quasi-variational inclusions, proximal-point mappings, $R$-accretive mappings, strongly accretive mappings, mixed Lipschitz continuous, $H$-Lipschitz continuous.

†Supported by NBHM, Department of Atomic Energy, Government of India under Grantsin-aid for Post-doctoral fellowship (Reference no. 40/2/2007-R\&D II/3910). 
In recent years, much attention has been given to develop general techniques for the sensitivity analysis of solution set of various classes of variational inequalities (inclusions). From the mathematical and engineering point of view, sensitivity properties of various classes of variational inequalities can be provide new insight concerning the problem being studied and can stimulate ideas for solving problems. The sensitivity analysis of solution set for variational inequalities have been studied extensively by many authors using quite different techniques. By using the projection technique, Defermos [11], Mukherjee and Verma [27], Noor [30] and Yen [37] studied the sensitivity analysis of solutions of some classes of variational inequalities with single-valued mappings. By using the implicit function approach that makes use of so-called normal mappings, Robinson [35] studied the sensitivity analysis of solutions for variational inequalities in finite-dimensional spaces. By using proximal-point mappings technique, Adly [1], Noor [35] and Agarwal et al. [2] studied the sensitivity analysis of solution set of some classes of quasi-variational inclusions involving single-valued mappings. By using projection and proximal-point mappings techniques, Ding and Luo [15], Liu et al. [26], Park and Jeong [32], Ding [14], and Kazmi and Khan [23, 24], studied the behavior and sensitivity analysis of solution set for some classes of generalized variational inequalities (inclusions) involving set-valued mappings. It is worth mentioning that most of the results in this direction have been obtained in the setting of Hilbert space.

Inspired by recent research work in this area, in this paper, we consider a system of parametric generalized quasi-variational inclusions (SPGQVI, for short) involving $R$-accretive mappings in uniformly smooth Banach space. Further, using $R$-proximal mappings technique of $R$-accretive mappings, and the properly of the fixed point set of set-valued mappings, we study the behavior and sensitivity analysis of the solution set for SPGQVI. Furthermore, the Lipschitz continuity of solution set of SPGQVI is proved under some suitable conditions. The theorems presented in this paper generalize and improve the results given by many authors, see for example [3, 22-24, 32-34].

\section{Preliminaries}

Let $E$ be a real Banach space equipped with norm $\|\cdot\|$; let $E^{*}$ be the topological dual space of $E$; let $\langle\cdot, \cdot\rangle$ is the dual pair of $E$ and $E^{*}$; let $C(E)$ denote the family of all nonempty compact subsets of $E$; let $2^{E}$ denote the power set of $E$; let $H(\cdot, \cdot)$ be the Hausdorff metric on $C(E)$ defined by

$$
H(A, B)=\max \left\{\sup _{x \in A} \inf _{y \in B} d(x, y), \sup _{y \in B} \inf _{x \in A} d(x, y)\right\}, A, B \in C(E),
$$

and let $J: E \rightarrow 2^{E^{*}}$ be the normalized duality mapping defined by

$$
J(x)=\left\{f \in E^{*}:\langle x, f\rangle=\|x\|^{2},\|x\|=\|f\|\right\}, x \in E .
$$

First, we recall and define the following concepts and results. 
Definition 2.1 ([9]). A Banach space $E$ is called smooth if, for every $x \in E$ with $\|x\|=1$, there exists a unique $f \in E^{*}$ such that $\|f\|=f(x)=1$. The modulus of smoothness of $E$ is the function $\rho_{E}:[0, \infty) \rightarrow[0, \infty)$, defined by

$$
\rho_{E}(\tau)=\sup \left\{\frac{(\|x+y\|+\|x-y\|)}{2}-1: x, y \in E,\|x\|=1,\|y\|=\tau\right\} .
$$

Definition 2.2 ([9]). The Banach space $E$ is said to be uniformly smooth, if

$$
\lim _{\tau \rightarrow 0} \frac{\rho_{E}(\tau)}{\tau}=0 .
$$

We note that if $E$ is smooth, then the normalized duality mapping $J$ is single-valued and if $E \equiv \mathcal{H}$, a Hilbert space, the $J$ is identity map on $\mathcal{H}$. In sequel, we denote a selection of normalized duality mapping by $j$.

Lemma 2.1 ([8, 21]). Let $E$ be an uniformly smooth Banach space and let $J: E \rightarrow E^{*}$ be the normalized duality mapping. Then for all $x, y \in E$, we have

(i) $\|x+y\|^{2} \leq\|x\|^{2}+2\langle y, J(x+y)\rangle$;

(ii) $\langle x-y, J(x)-J(y)\rangle \leq 2 d^{2} \rho_{E}(4\|x-y\| / d)$, where $d=\sqrt{\left(\|x\|^{2}+\|y\|^{2}\right) / 2}$.

Definition 2.3 ([9]). A mapping $A: E \rightarrow E$ is said to be:

(i) accretive if, for all $x, y \in E$, there exists $j(x-y) \in J(x-y)$ such that

$$
\langle A(x)-A(y), j(x-y)\rangle \geq 0 ;
$$

(ii) strictly accretive if, $\langle A(x)-A(y), j(x-y)\rangle>0$, and the equality holds only when $x=y$.

(iii) $\xi$-strongly accretive if, for all $x, y \in E$, there exist $j(x-y) \in J(x-y)$ and a constant $\xi>0$ such that

$$
\langle A(x)-A(y), j(x-y)\rangle \geq \xi\|x-y\|^{2} ;
$$

(iv) $\delta$-Lipschitz continuous if, for all $x, y \in E$, there exists a constant $\delta>0$ such that

$$
\|A(x)-A(y)\| \leq \delta\|x-y\| .
$$

Definition 2.4 ([8]). A set-valued mapping $M: E \rightarrow 2^{E}$ is said to be:

(i) accretive if, for all $x, y \in E$, there exist $j(x-y) \in J(x-y)$ such that

$$
\langle u-v, j(x-y)\rangle \geq 0 \text { for all } u \in M(x), v \in M(y) ;
$$

(ii) $\xi$-strongly accretive if, for all $x, y \in E$, there exist $j(x-y) \in J(x-y)$ and a constant $\xi>0$ such that

$$
\langle u-v, j(x-y)\rangle \geq \xi\|x-y\|^{2} \text { for all } u \in M(x), v \in M(y) ;
$$

(iii) $m$-accretive if, $M$ is accretive and $(I+\rho M)(E)=E$ for any $\rho>0$, where $I$ stands for identity mapping.

Definition 2.5 ([16]). Let $R: E \rightarrow E$ be a nonlinear mapping. Then a setvalued mapping $M: E \rightarrow 2^{E}$ is said to be $R$-accretive, if $M$ is accretive and $(R+\rho M)(E)=E$ for any $\rho>0$. 
The following result gives some properties of $R$-accretive mappings:

Lemma 2.2 ([16]). Let $R: E \rightarrow E$ be a strictly accretive mapping and let $M: E \rightarrow 2^{E}$ be a $R$-accretive set-valued mapping. Then

(i) $\langle u-v, J(x-y)\rangle \geq 0$ for all $(v, y) \in \operatorname{Graph}(M)$ implies $(u, x) \in$ $\operatorname{Graph}(M)$, where $\operatorname{Graph}(M):=\{u, x) \in E \times E: u \in M(x)\} ;$

(ii) the mapping $(R+\rho M)^{-1}$ is single-valued for all $\rho>0$.

By Lemma 2.2, we can define $R$-proximal point mapping of a $R$-accretive mapping $M$ as follows

$$
J_{\rho}^{M}(z)=(R+\rho M)^{-1}(z) \text { for all } z \in E,
$$

where $\rho>0$ is a constant and $R: E \rightarrow E$ is a strictly accretive mapping.

Lemma 2.3. Let $R: E \rightarrow E$ be a $\gamma$-strongly accretive mapping and $M: E \rightarrow$ $2^{E}$ be a $R$-accretive mapping. Then the $R$-proximal-point mapping $J_{\rho}^{M}: E \rightarrow E$ is $\frac{1}{\gamma}$-Lipschitz continuous, that is,

$$
\left\|J_{\rho}^{M}(x)-J_{\rho}^{M}(y)\right\| \leq \frac{1}{\gamma}\|x-y\| \text { for all } x, y \in E .
$$

Lemma 2.4 ([25]). Let $X$ be a complete metric space and let $T_{1}, T_{2}: X \rightarrow$ $C(X)$ be $\theta-H$-contraction mappings. Then

$$
H\left(F\left(T_{1}\right), F\left(T_{2}\right)\right) \leq(1-\theta)^{-1} \sup _{x \in X}\left(T_{1}(x), T_{2}(x)\right),
$$

where $F\left(T_{1}\right)$ and $F\left(T_{2}\right)$ are the set of fixed points of $T_{1}$ and $T_{2}$ respectively.

\section{System of parametric generalized quasi-variational inclusions}

Throughout rest of this paper, unless otherwise stated, for each $i=1,2$, we assume that $E_{i}$ is uniformly smooth Banach space with norm $\|\cdot\|_{i}$, and denote the duality pairing between $E_{i}$ and its dual $E_{i}^{*}$ by $\langle\cdot, \cdot\rangle_{i}$.

Let $\Omega_{1}$ and $\Omega_{2}$ be nonempty subsets of $E_{1}$ and $E_{2}$, respectively, in which parameters $\lambda$ and $\mu$ takes the values; let $R_{i}: E_{i} \rightarrow E_{i} ; g_{i}: E_{i} \times \Omega_{i} \rightarrow E_{i}$; $P, F: E_{1} \times E_{2} \times \Omega_{1} \times \Omega_{2} \rightarrow E_{1}$ and let $Q, G: E_{1} \times E_{2} \times \Omega_{1} \times \Omega_{2} \rightarrow E_{2}$ be singlevalued mappings such that $g \neq 0$ and let $A, C: E_{1} \times \Omega_{1} \rightarrow C\left(E_{1}\right)$ and $B, D$ : $E_{2} \times \Omega_{2} \rightarrow C\left(E_{2}\right)$ be set-valued mappings. Let $M: E_{1} \times \Omega_{1} \rightarrow 2^{E_{1}}$ be an $R_{1}$ accretive mapping and $N: E_{2} \times \Omega_{2} \rightarrow 2^{E_{2}}$ be an $R_{2}$-accretive mapping in the first argument such that $g_{1}(x, \lambda) \in \operatorname{dom} M(\cdot, \lambda)$ and $g_{2}(y, \mu) \in \operatorname{dom} N(\cdot, \mu)$ for all $x \in E_{1}, y \in E_{2}, \lambda \in \Omega_{1}, \mu \in \Omega_{2}$. For each $\left(f_{1}, f_{2}, \lambda, \mu\right) \in E_{1} \times E_{2} \times \Omega_{1} \times \Omega_{2}$, we consider the system of parametric generalized quasi-variational inclusions (SPGQVI, for short):

Find $(x, y, u, v, w, z)$ such that $(x=x(\lambda), y=y(\mu)) \in E_{1} \times E_{2}, u=u(\lambda) \in$ $A(x, \lambda), v=v(\mu) \in B(y, \mu), w=w(\lambda) \in C(x, \lambda), z=z(\mu) \in D(y, \mu)$, and

$$
\left\{\begin{array}{l}
f_{1} \in F(x, y, \lambda, \mu)+P(u, v, \lambda, \mu)+M\left(g_{1}(x, \lambda), \lambda\right) \\
f_{2} \in G(x, y, \lambda, \mu)+Q(w, z, \lambda, \mu)+N\left(g_{2}(y, \mu), \mu\right)
\end{array}\right.
$$




\section{Some special cases of SPGQVI (3.1):}

If $E_{1} \equiv E_{2} \equiv H ; F \equiv G ; R_{1} \equiv R_{2} ; f_{1} \equiv f_{2} \equiv 0 ; P \equiv Q \equiv 0 ; B \equiv D \equiv 0 ; g=$ $g_{1} \equiv g_{2} ; C \equiv 0 ; F(x, y, \lambda, \mu) \equiv F(x, \lambda)$ for all $(x, y, \lambda, \mu) \in E_{1} \times E_{2} \times \Omega_{1} \times \Omega_{2}$, then SPGQVLI (3.1) reduces to the problem of finding $x=x(\lambda) \in H$ such that

$$
0 \in F(x, \lambda)+M(g(x, \lambda), \lambda),
$$

which has been studied by Adly [1].

Now, for each fixed $(\lambda, \mu) \in \Omega_{1} \times \Omega_{2}$, the solution set $S(\lambda, \mu)$ of SPGMQVI(3.1) is defined as

$$
\begin{aligned}
& S(\lambda, \mu):=\left\{\begin{array}{l}
(x=x(\lambda), y=y(\mu)) \in E_{1} \times E_{2}, u=u(\lambda) \in A(x, \lambda), \\
v=v(\mu) \in B(y, \mu), w=w(\lambda) \in C(x, \lambda), z=z(\mu) \in D(y, \mu), \\
\text { such that } \\
f_{1} \in F(x, y, \lambda, \mu)+P(u, v, \lambda, \mu)+M\left(g_{1}(x, \lambda), \lambda\right) \\
f_{2} \in G(x, y, \lambda, \mu)+Q(w, z, \lambda, \mu)+N\left(g_{2}(y, \mu), \mu\right)
\end{array}\right\} .
\end{aligned}
$$

The aim of this paper is to study the behavior and sensitivity analysis of the solution set $S(\lambda, \mu)$, and the conditions on mappings $A, B, C, D, F, G, P, Q$, $M, N, g_{i}, R_{i}$; under which the solution set $S(\lambda, \mu)$ of SPGQVI(3.1) is nonempty and Lipschitz continuous with respect to the parameters $(\lambda, \mu) \in \Omega_{1} \times \Omega_{2}$.

First, we recall the following concepts:

Definition 3.1 ([22]). A mapping $g_{1}: E_{1} \times \Omega_{1} \rightarrow E_{1}$ is said to be:

(i) $\left(L_{g_{1}}, l_{g_{1}}\right)$-mixed Lipschitz continuous, if there exist constants $L_{g_{1}}, l_{g_{1}}$ $>0$ such that $\left\|g_{1}\left(x_{1}, \lambda_{1}\right)-g_{1}\left(x_{2}, \lambda_{2}\right)\right\|_{1}$

$\leq L_{g_{1}}\left\|x_{1}-x_{2}\right\|_{1}+l_{g_{1}}\left\|\lambda_{1}-\lambda_{2}\right\|$ for all $\left(x_{1}, \lambda_{1}\right),\left(x_{2}, \lambda_{2}\right) \in E_{1} \times \Omega_{1}$,

(ii) $s_{1}$-strongly accretive, if there exists a constant $s_{1}>0$ such that

$$
\left\langle g_{1}\left(x_{1}, \lambda\right)-g_{1}\left(x_{2}, \lambda\right), J_{1}\left(x_{1}-x_{2}\right)\right\rangle_{1} \geq s_{1}\left\|x_{1}-x_{2}\right\|_{1}^{2}
$$

for all $\left(x_{1}, x_{2}, \lambda\right) \in E_{1} \times E_{2} \times \Omega_{1}$, where $J_{1}: E_{1} \rightarrow 2^{E_{1}^{*}}$ is the normalized duality mapping.

Remark 3.1. When $\lambda$ is fixed, then mixed Lipschitz continuity of $g_{1}$ implies Lipschitz continuity in the first argument.

Definition 3.2 ([22]). A set-valued mapping $A: E_{1} \times \Omega_{1} \rightarrow C\left(E_{1}\right)$ is said to be $\left(L_{A}, l_{A}\right)$-H-mixed Lipschitz continuous, if there exist constants $L_{A}, l_{A}>0$ such that

$$
\begin{aligned}
& H\left(A\left(x_{1}, \lambda_{1}\right), A\left(x_{2}, \lambda_{2}\right)\right) \\
\leq & L_{A}\left\|x_{1}-x_{2}\right\|_{1}+l_{A}\left\|\lambda_{1}-\lambda_{2}\right\|_{1} \text { for all }\left(x_{1}, \lambda_{1}\right),\left(x_{2}, \lambda_{2}\right) \in E_{1} \times \Omega_{1} .
\end{aligned}
$$

Definition 3.3 ([22]). Let $R_{1}: E_{1} \rightarrow E_{1}, g_{1}: E_{1} \times \Omega_{1} \rightarrow E_{1}$. Then a mapping $F: E_{1} \times E_{2} \times \Omega_{1} \times \Omega_{2} \rightarrow E_{1}$ is said to be: 
(i) $\left(L_{(F, 1)}, L_{(F, 2)}, l_{(F, 1)}, l_{(F, 2)}\right)$-mixed Lipschitz continuous, if there exist constants $L_{(F, 1)}, L_{(F, 2)}, L_{(F, 1)}, L_{(F, 2)}>0$ such that

$\left\|F\left(x_{1}, y_{1}, \lambda_{1}, \mu_{1}\right)-F\left(x_{2}, y_{2}, \lambda_{2}, \mu_{2}\right)\right\|_{1}$

$\leq L_{(F, 1)}\left\|x_{1}-x_{2}\right\|_{1}+L_{(F, 2)}\left\|y_{1}-y_{2}\right\|_{2}+l_{(F, 1)}\left\|\lambda_{1}-\lambda_{2}\right\|_{1}+l_{(F, 2)}\left\|\mu_{1}-\mu_{2}\right\|_{2}$

for all $\left(x_{1}, y_{1}, \lambda_{1}, \mu_{1}\right),\left(x_{2}, y_{2}, \lambda_{2}, \mu_{2}\right) \in E_{1} \times E_{2} \times \Omega_{1} \times \Omega_{2}$;

(ii) $\xi_{1}$-strongly $R_{1} \circ g_{1}$-accretive in the first argument if there exists a constant $\xi_{1}>0$ such that

$\left\langle F\left(x_{1}, y_{1}, \lambda, \mu\right)-F\left(x_{2}, y_{1}, \lambda, \mu\right), J_{1}\left(R_{1} \circ g_{1}\left(x_{1}, \lambda\right)-R_{1} \circ g_{1}\left(x_{2}, \lambda\right)\right)\right\rangle$

$\geq \xi_{1}\left\|x_{1}-x_{2}\right\|_{1}^{2}$

for all $x_{1}, x_{2} \in E_{1}, y_{1} \in E_{2},(\lambda, \mu) \in \Omega_{1} \times \Omega_{2}$, where $R_{1} \circ g_{1}$ denote $R_{1}$ composition $g_{1}$.

We now transfer the SPGQVI (3.1) into a fixed point problem.

Lemma 3.1. For each $\left(f_{1}, f_{2}, \lambda, \mu\right) \in E_{1} \times E_{2} \times \Omega_{1} \times \Omega_{2},(x, y, u, v, w, z)$ with $(x=x(\lambda), y=y(\mu)) \in E_{1} \times E_{2}, u=u(\lambda) \in A(x, \lambda), v=v(\mu) \in B(y, \mu)$, $w=w(\lambda) \in C(x, \lambda), z=z(\mu) \in D(y, \mu)$ is a solution of SPGQVI (3.1) if and only if the set-valued mapping $T: E_{1} \times E_{2} \times \Omega_{1} \times \Omega_{2} \rightarrow 2^{E_{1} \times E_{2}}$ defined by

$$
T(x, y, \lambda, \mu)=(U(x, y, \lambda, \mu), V(x, y, \lambda, \mu)),
$$

where $U: E_{1} \times E_{2} \times \Omega_{1} \times \Omega_{2} \rightarrow 2^{E_{1}}$ and $V: E_{1} \times E_{2} \times \Omega_{1} \times \Omega_{2} \rightarrow 2^{E_{2}}$ are defined as

$$
\begin{aligned}
& U(x, y, \lambda, \mu) \\
& =\bigcup_{u \in A(x, \lambda), v \in B(y, \mu)}\left[x-g_{1}(x, \lambda)+J_{\rho_{1}}^{M(\cdot, \lambda)}\left(R_{1} \circ g_{1}(x, \lambda)-\rho_{1} F(x, y, \lambda, \mu)\right.\right. \\
& \left.\left.-\rho_{1} P(u, v, \lambda, \mu)+\rho_{1} f_{1}\right)\right],
\end{aligned}
$$

$$
\begin{aligned}
& =\bigcup_{w \in C(x, \lambda), z \in D(y, \mu)}^{V(x, y, \lambda, \mu)}\left[y-g_{2}(y, \mu)+J_{\rho_{2}}^{N(\cdot, \mu)}\left(R_{2} \circ g_{2}(y, \mu)-\rho_{2} G(x, y, \lambda, \mu)\right.\right. \\
& \left.\left.\quad-\rho_{2} Q(w, z, \lambda, \mu)+\rho_{2} f_{2}\right)\right],
\end{aligned}
$$

has a fixed point, where $J_{\rho_{1}}^{M(\cdot, \lambda)}=\left(R_{1}+\rho_{1} M(\cdot, \lambda)\right)^{-1}, J_{\rho_{2}}^{N(\cdot, \mu)}=\left(R_{2}+\right.$ $\left.\rho_{2} N(\cdot, \mu)\right)^{-1}$ and $\rho_{1}, \rho_{2}>0$ are constants.

Proof. For each $\left(f_{1}, f_{2}, \lambda, \mu\right) \in E_{1} \times E_{2} \times \Omega_{1} \times \Omega_{2}$, SPGQVI (3.1) has a solution $(x, y, u, v, w, z)$ with $\left(x=x(\lambda) \in E_{1}, y=y(\mu) \in E_{2}, u=u(x, \lambda) \in A(x, \lambda)\right.$, $v=v(\mu) \in B(y, \mu), w=w(\lambda) \in C(x, \lambda), z=z(\mu) \in D(y, \mu)$ such that 
$g_{1}(x, \lambda) \in \operatorname{dom} M(\cdot, \lambda)$ and $g_{2}(y, \mu) \in \operatorname{dom} N(\cdot, \mu)$ for all $x \in E_{1}, y \in E_{2}, \lambda \in$ $\Omega_{1}, \mu \in \Omega_{2}$ if and only if

$$
\begin{aligned}
& f_{1} \in F(x, y, \lambda, \mu)-P(u, v, \lambda, \mu)+M\left(g_{1}(x, \lambda), \lambda\right) \\
\Longleftrightarrow & R_{1} \circ g_{1}(x, \lambda)-\rho_{1} F(x, y, \lambda, \mu)+\rho_{1} P(u, v, \lambda, \mu)+\rho_{1} f_{1} \\
& \in\left(R_{1}+\rho_{1} M(\cdot, \lambda)\right)\left(g_{1}(x, \lambda)\right)
\end{aligned}
$$

and

$$
\begin{aligned}
& f_{2} \in G(x, y, \lambda, \mu)-Q(w, z, \lambda, \mu)+N\left(g_{2}(y, \mu), \mu\right) \\
\Longleftrightarrow & R_{2} \circ g_{2}(y, \mu)-\rho_{2} G(x, y, \lambda, \mu)+\rho_{2} Q(w, z, \lambda, \mu)+\rho_{2} f_{2} \\
& \in\left(R_{2}+\rho_{2} N(\cdot, \mu)\right)\left(g_{2}(y, \mu)\right) .
\end{aligned}
$$

Since for each $\left.x \in E_{1}, y \in E_{2}, \lambda \in \Omega_{1}, \mu\right) \in \Omega_{2}, M(\cdot, \lambda)$ is $R_{1}$-accretive and $N(\cdot, \mu)$ is $R_{2}$-accretive, by definition of $R_{i}$-proximal-point mapping of $J_{\rho_{1}}^{M(\cdot, \lambda)}$ and $J_{\rho_{2}}^{M(\cdot, \mu)}$ preceding inclusions hold if and only if

$$
g_{1}(x, \lambda)=J_{\rho_{1}}^{M(\cdot, \lambda)}\left[R_{1} \circ g_{1}(x, \lambda)-\rho_{1} F(x, y, \lambda, \mu)+\rho_{1} P(u, v, \lambda, \mu)+\rho_{1} f_{1}\right]
$$

and

$$
g_{2}(y, \mu)=J_{\rho_{2}}^{N(\cdot, \mu)}\left[R_{2} \circ g_{2}(y, \mu)-\rho_{2} G(x, y, \lambda, \mu)+\rho_{2} Q(u, v, \lambda, \mu)+\rho_{2} f_{2}\right],
$$

i.e., $(x, y) \in T(x, y, \lambda, \mu)$. This completes the proof.

\section{Sensitivity analysis of solution set $S(\lambda, \mu)$}

Now, we shall study the behavior and sensitivity analysis of the solution set of SPGMQVI (3.1) and further, under suitable conditions, we shall discuss Lipschitz continuity of the solution set with respect to the parameters.

Theorem 4.1. For each $i=1,2$, let $E_{i}$ be uniformly smooth Banach space with $\rho_{E_{i}}(t) \leq c_{i} t^{2}$ for some $c_{i}>0$; let the mappings $R_{i}: E_{i} \rightarrow E_{i}$ and $g_{i}: E_{i} \times \Omega_{i} \rightarrow$ $E_{i}$ such that $g_{i}$ is $s_{i}$-strongly accretive and $\left(L_{g_{i}}, l_{g_{i}}\right)$-mixed Lipschitz continuous and $R_{i} \circ g_{i}$ be $\left(L_{R_{i} \circ g_{i}}, l_{R_{i} \circ g_{i}}\right)$-mixed Lipschitz continuous; let the set-valued mappings $A, C: E_{1} \times \Omega_{1} \rightarrow C\left(E_{1}\right)$ and $B, D: E_{2} \times \Omega_{2} \rightarrow C\left(E_{2}\right)$ be $H$-mixed Lipschitz continuous with constants $\left(L_{A}, l_{A}\right),\left(L_{B}, l_{B}\right)$ and $\left(L_{C}, l_{C}\right),\left(L_{D}, l_{D}\right)$, respectively; let $F: E_{1} \times E_{2} \times \Omega_{1} \times \Omega_{2} \rightarrow E_{1}$ be $\xi_{1}$-strongly accretive with respect to $R_{1} \circ g_{1}$ in the first argument and $\left(L_{(F, 1)}, L_{(F, 2)}, l_{(F, 1)}, l_{(F, 2)}\right)$-mixed Lipschitz continuous and $G: E_{1} \times E_{2} \times \Omega_{1} \times \Omega_{2} \rightarrow E_{2}$ be $\xi_{2}$-strongly accretive with respect to $R_{2} \circ g_{2}$ in the second argument and $\left(L_{(G, 1)}, L_{(G, 2)}, l_{(G, 1)}, l_{(G, 2)}\right)$ mixed Lipschitz continuous; let the mappings $P: E_{1} \times E_{2} \times \Omega_{1} \times \Omega_{2} \rightarrow$ $E_{1}$ be $\left(L_{(P, 1)}, L_{(P, 2)}, l_{(P, 1)}, l_{(P, 2)}\right)$-mixed Lipschitz continuous and $Q: E_{1} \times$ $E_{2} \times \Omega_{1} \times \Omega_{2} \rightarrow E_{2}$ be $\left(L_{(Q, 1)}, L_{(Q, 2)}, l_{(Q, 1)}, l_{(Q, 2)}\right)$-mixed Lipschitz continuous, respectively; let $M: E_{1} \times \Omega_{1} \rightarrow 2^{E_{1}}$ and $N: E_{2} \times \Omega_{2} \rightarrow 2^{E_{2}}$ be such that for each fixed $\lambda \in \Omega_{1}$ and $\mu \in \Omega_{2}, M(\cdot, \lambda)$ and $N(\cdot, \mu)$ are $R_{1}$-accretive and 
$R_{2}$-accretive mappings, respectively. Suppose that there are constants $\delta_{1}, \delta_{2}>0$ satisfies the following condition:

(4.1)

$$
\left\{\begin{aligned}
k_{1}:= & \sqrt{1-2 s_{2}+64 c_{1} L_{g_{1}}^{2}}+\frac{\rho_{1}}{\gamma_{1}} L_{(P, 1)} L_{A}+\frac{1}{\gamma_{1}} \sqrt{L_{R_{1} \circ g_{1}}^{2}-2 \rho_{1} \xi_{1}+64 c_{1} \rho_{1}^{2} L_{(F, 1)}^{2}} \\
& +\frac{\rho_{2}}{\gamma_{2}}\left(L_{(G, 1)}+L_{(Q, 1)} L_{C}\right)<1 \\
k_{2}:= & \sqrt{1-2 s_{2}+64 c_{2} L_{g_{2}}^{2}}+\frac{\rho_{2}}{\gamma_{2}} L_{(Q, 2)} L_{D}+\frac{1}{\gamma_{2}} \sqrt{L_{R_{2} \circ g_{2}}^{2}-2 \rho_{2} \xi_{2}+64 c_{2} \rho_{2}^{2} L_{(G, 2)}^{2}} \\
& +\frac{\rho_{1}}{\gamma_{1}}\left(L_{(F, 2)}+L_{(P, 2)} L_{B}\right)<1,
\end{aligned}\right.
$$

where $\theta=\max \left\{k_{1}, k_{2}\right\}$. Then for each fixed $\left(f_{1}, f_{2}\right) \in E_{1} \times E_{2}$, the set-valued mapping $T$ defined by (3.3) is a compact-valued uniform $\theta-H$-contraction mapping with respect to $(\lambda, \mu) \in \Omega_{1} \times \Omega_{2}$, where $\theta$ is given by (4.1). Moreover, for each $(\lambda, \mu) \in \Omega_{1} \times \Omega_{2}$, the solution set $S(\lambda, \mu)$ of SPGQVI (3.1) is nonempty and closed.

Proof. Let $(x, y, \lambda, \mu)$ be an arbitrary element in $E_{1} \times E_{2} \times \Omega_{1} \times \Omega_{2}$. Since $A, B, C, D$ are compact-valued, then for any sequences $\left\{u_{n}\right\} \subset A(x, \lambda),\left\{v_{n}\right\} \subset$ $B(y, \mu),\left\{w_{n}\right\} \subset C(x, \lambda),\left\{z_{n}\right\} \subset D(y, \mu)$, there exist subsequences $\left\{u_{n_{i}}\right\} \subset$ $\left\{u_{n}\right\},\left\{v_{n_{i}}\right\} \subset\left\{v_{n}\right\},\left\{w_{n_{i}}\right\} \subset\left\{w_{n}\right\},\left\{z_{n_{i}}\right\} \subset\left\{z_{n}\right\}$ such that $u_{n_{i}} \rightarrow u, v_{n_{i}} \rightarrow v$, $w_{n_{i}} \rightarrow w, z_{n_{i}} \rightarrow z$ as $i \rightarrow \infty$. By using Lemma 2.3 and mixed Lipschitz continuity of $P$ and $Q$, we have

$$
\begin{aligned}
& \| J_{\rho_{1}}^{M(\cdot, \lambda)}\left[R_{1} \circ g_{1}(x, \lambda)-\rho_{1} F(x, y, \lambda, \mu)-\rho_{1} P\left(u_{n_{i}}, v_{n_{i}}, \lambda, \mu\right)+\rho_{1} f_{1}\right] \\
& \quad-J_{\rho_{1}}^{M(\cdot, \lambda)}\left[R_{1} \circ g_{1}(x, \lambda)-\rho_{1} F(x, y, \lambda, \mu)-\rho_{1} P(u, v, \lambda, \mu)+\rho_{1} f_{1}\right] \|_{1} \\
\leq & \frac{\rho_{1}}{\gamma_{1}}\left\|P\left(u_{n_{i}}, v_{n_{i}}, \lambda, \mu\right)-P(u, v, \lambda, \mu)\right\|_{1} \\
\leq & \frac{\rho_{1}}{\gamma_{1}}\left[L_{(P, 1)}\left\|u_{n_{i}}-u\right\|_{1}+L_{(P, 2)}\left\|v_{n_{i}}-v\right\|_{2}\right] \rightarrow 0 \text { as } i \rightarrow \infty
\end{aligned}
$$

and

$$
\begin{aligned}
& \| J_{\rho_{2}}^{N(\cdot, \mu)}\left[R_{2} \circ g_{2}(y, \mu)-\rho_{2} G(x, y, \lambda, \mu)-\rho_{2} Q\left(w_{n_{i}}, z_{n_{i}}, \lambda, \mu\right)+\rho_{2} f_{2}\right] \\
& \quad-J_{\rho_{2}}^{N(\cdot, \mu)}\left[R_{2} \circ g_{2}(y, \mu)-\rho_{2} G(x, y, \lambda, \mu)-\rho_{2} Q(w, z, \lambda, \mu)+\rho_{2} f_{2}\right] \|_{2} \\
\leq & \frac{\rho_{2}}{\gamma_{2}}\left\|Q\left(w_{n_{i}}, z_{n_{i}}, \lambda, \mu\right)-Q(w, z, \lambda, \mu)\right\|_{2} \\
\leq & \frac{\rho_{2}}{\gamma_{2}}\left[L_{(Q, 1)}\left\|w_{n_{i}}-u\right\|_{1}+L_{(Q, 2)}\left\|z_{n_{i}}-z\right\|_{2}\right] \rightarrow 0 \text { as } i \rightarrow \infty .
\end{aligned}
$$

Thus (3.3), (4.2) and (4.3) yield that $T(x, y, \lambda, \mu) \in C\left(E_{1} \times E_{2}\right)$.

Now, for each fixed $(\lambda, \mu) \in \Omega_{1} \times \Omega_{2}$, we prove that $T(x, y, \lambda, \mu)$ is a uniform $\theta$-H-contraction mapping. Let for $i=1,2,\left(x_{i}, y_{i}, \lambda, \mu\right)$ be arbitrary elements in $E_{1} \times E_{2} \times \Omega_{1} \times \Omega_{2}$ and let $t_{1} \in U\left(x_{1}, y_{1}, \lambda, \mu\right)$ and $p_{1} \in V\left(x_{1}, y_{1}, \lambda, \mu\right)$, there 
exist $u_{1}=u_{1}\left(x_{1}, \lambda\right) \in A\left(x_{1}, \lambda\right), v_{1}=v_{1}\left(y_{1}, \mu\right) \in B\left(y_{1}, \mu\right), w_{1}=w_{1}\left(x_{1}, \lambda\right) \in$ $C\left(x_{1}, \lambda\right)$, and $z_{1}=z_{1}\left(y_{1}, \mu\right) \in D\left(y_{1}, \mu\right)$ such that

$(4.4)$

$$
\begin{aligned}
t_{1}= & x_{1}-g_{1}\left(x_{1}, \lambda\right) \\
& +J_{\rho_{1}}^{M(\cdot, \lambda)}\left[R_{1} \circ g_{1}\left(x_{1}, \lambda\right)-\rho_{1} F\left(x_{1}, y_{1}, \lambda, \mu\right)+\rho_{1} P\left(u_{1}, v_{1}, \lambda, \mu\right)+\rho_{1} f_{1}\right]
\end{aligned}
$$

and

(4.5)

$$
\begin{aligned}
p_{1}= & y_{1}-g_{2}\left(y_{1}, \mu\right) \\
& +J_{\rho_{2}}^{N(\cdot, \mu)}\left[R_{2} \circ g_{2}\left(y_{1}, \mu\right)-\rho_{2} G\left(x_{1}, y_{1}, \lambda, \mu\right)+\rho_{2} Q\left(w_{1}, z_{1}, \lambda, \mu\right)+\rho_{2} f_{2}\right] .
\end{aligned}
$$

It follows from the compactness of $A\left(x_{2}, \lambda\right), B\left(y_{2}, \mu\right), C\left(x_{2}, \lambda\right)$ and $D\left(y_{2}, \mu\right)$ and $H$-Lipschitz continuity of $A, B, C, D$ that there exist $u_{2}=u_{2}\left(x_{2}, \lambda\right) \in A\left(x_{2}, \lambda\right)$, $v_{2}=v_{2}\left(y_{2}, \mu\right) \in B\left(y_{2}, \mu\right), w_{2}=w_{2}\left(x_{2}, \lambda\right) \in C\left(x_{2}, \lambda\right)$ and $z_{2}=z_{2}\left(y_{2}, \mu\right) \in$ $D\left(y_{2}, \mu\right)$ such that

$$
\begin{aligned}
\left\|u_{1}-u_{2}\right\|_{1} & \leq H\left(A\left(x_{1}, \lambda\right), A\left(x_{2}, \lambda\right)\right) \leq L_{A}\left\|x_{1}-x_{2}\right\|_{1}, \\
\left\|v_{1}-v_{2}\right\|_{2} & \leq H\left(B\left(y_{1}, \mu\right), B\left(y_{2}, \mu\right)\right) \leq L_{B}\left\|y_{1}-y_{2}\right\|_{2}, \\
\left\|w_{1}-w_{2}\right\|_{1} \leq H\left(C\left(x_{1}, \lambda\right), C\left(x_{2}, \lambda\right)\right) & \leq L_{C}\left\|x_{1}-x_{2}\right\|_{1}, \\
\left\|z_{1}-z_{2}\right\|_{2} & \leq H\left(D\left(y_{1}, \mu\right), D\left(y_{2}, \mu\right)\right) \leq L_{D}\left\|y_{1}-y_{2}\right\|_{2} .
\end{aligned}
$$

Let

$$
\begin{aligned}
t_{2}=x_{2} & -g_{1}\left(x_{2}, \lambda\right) \\
& +J_{\rho_{1}}^{M(\cdot, \lambda)}\left[R_{1} \circ g_{1}\left(x_{2}, \lambda\right)-\rho_{1} F\left(x_{2}, y_{2}, \lambda, \mu\right)+\rho_{1} P\left(u_{2}, v_{2}, \lambda, \mu\right)+\rho_{1} f_{1}\right]
\end{aligned}
$$

and

(4.8)

$$
\begin{aligned}
p_{2}=y_{2} & -g_{2}\left(y_{2}, \mu\right) \\
& +J_{\rho_{2}}^{N(\cdot, \mu)}\left[R_{2} \circ g_{2}\left(y_{2}, \mu\right)-\rho_{2} G\left(x_{2}, y_{2}, \lambda, \mu\right)+\rho_{2} Q\left(w_{2}, z_{2}, \lambda, \mu\right)+\rho_{2} f_{2}\right] .
\end{aligned}
$$

Then we have $t_{2} \in U\left(x_{2}, y_{2}, \lambda, \mu\right)$ and $p_{2} \in V\left(x_{2}, y_{2}, \lambda, \mu\right)$.

Next, using Lemma 2.3, we have

$$
\begin{aligned}
& \left\|t_{1}-t_{2}\right\|_{1} \\
\leq & \left\|x_{1}-x_{2}-\left(g_{1}\left(x_{1}, \lambda\right)-g_{1}\left(x_{2}, \lambda\right)\right)\right\|_{1} \\
& +\| J_{\rho_{1}}^{M(\cdot, \lambda)}\left[R_{1} \circ g_{1}\left(x_{1}, \lambda\right)-\rho_{1} F\left(x_{1}, y_{1}, \lambda, \mu\right)+\rho_{1} P\left(u_{1}, v_{1}, \lambda, \mu\right)+\rho_{1} f_{1}\right] \\
& -J_{\rho_{1}}^{M(\cdot, \lambda)}\left[R_{1} \circ g_{1}\left(x_{2}, \lambda\right)-\rho_{1} F\left(x_{2}, y_{2}, \lambda, \mu\right)+\rho_{1} P\left(u_{2}, v_{2}, \lambda, \mu\right)+\rho_{1} f_{1}\right] \|_{1} \\
\leq & \left\|x_{1}-x_{2}-\left(g_{1}\left(x_{1}, \lambda\right)-g_{1}\left(x_{2}, \lambda\right)\right)\right\|_{1} \\
& +\frac{1}{\gamma_{1}} \| R_{1} \circ g_{1}\left(x_{1}, \lambda\right)-R_{1} \circ g_{1}\left(x_{2}, \lambda\right)-\rho_{1}\left(F\left(x_{1}, y_{1}, \lambda, \mu\right)-F\left(x_{2}, y_{2}, \lambda, \mu\right)\right. \\
& \left.+P\left(u_{1}, v_{1}, \lambda, \mu\right)-P\left(u_{2}, v_{2}, \lambda, \mu\right)\right) \|_{1} .
\end{aligned}
$$


Since $g_{1}$ is $s_{1}$-strongly accretive and $\left(L_{g_{1}}, l_{g_{1}}\right)$-mixed Lipschitz continuous, using Lemma 2.1 we have

$$
\begin{aligned}
& \left\|x_{1}-x_{2}-\left(g_{1}\left(x_{1}, \lambda\right)-g_{1}\left(x_{2}, \lambda\right)\right)\right\|_{1}^{2} \\
\leq & \left\|x_{1}-x_{2}\right\|_{1}^{2}-2\left\langle g_{1}\left(x_{1}, \lambda\right)-g_{1}\left(x_{2}, \lambda\right), J_{1}\left(x_{1}-x_{2}-\left(g_{1}\left(x_{1}, \lambda\right)-g_{1}\left(x_{2}, \lambda\right)\right)\right)\right\rangle_{1} \\
\leq & \left\|x_{1}-x_{2}\right\|_{1}^{2}-2\left\langle g_{1}\left(x_{1}, \lambda\right)-g_{1}\left(x_{2}, \lambda\right), J_{1}\left(x_{1}-x_{2}\right\rangle_{1}-2\left\langle g_{1}\left(x_{1}, \lambda\right)-g_{1}\left(x_{2}, \lambda\right),\right.\right. \\
& \left.J_{1}\left(x_{1}-x_{2}-\left(g_{1}\left(x_{1}, \lambda\right)-g_{1}\left(x_{2}, \lambda\right)\right)\right)-J_{1}\left(x_{1}-x_{2}\right)\right\rangle_{1} \\
\leq & \left\|x_{1}-x_{2}\right\|_{1}^{2}-2 s_{1}\left\|x_{1}-x_{2}\right\|_{1}^{2}+64 c_{1} L_{g_{1}}^{2}\left\|x_{1}-x_{2}\right\|_{1}^{2} \\
\leq & \left(1-2 s_{1}+64 c_{1} L_{g_{1}}^{2}\right)\left\|x_{1}-x_{2}\right\|_{1}^{2} .
\end{aligned}
$$

Since $P$ is $\left(L_{(P, 1)}, L_{(P, 2)}, l_{(P, 1)}, l_{(P, 2)}\right)$-mixed Lipschitz continuous and $H$ Lipschitz continuity of set-valued mappings $A, B$, we have

$$
\begin{aligned}
& \left\|P\left(u_{1}, v_{1}, \lambda_{1}, \mu_{1}\right)-P\left(u_{2}, v_{2}, \lambda_{2}, \mu_{2}\right)\right\|_{1} \\
\leq & L_{(P, 1)}\left\|u_{1}-u_{2}\right\|_{1}+L_{(P, 2)}\left\|v_{1}-v_{2}\right\|_{2}+l_{(P, 1)}\left\|\lambda_{1}-\lambda_{2}\right\|_{1}+l_{(P, 2)}\left\|\mu_{1}-\mu_{2}\right\|_{2} \\
\leq & L_{(P, 1)} H\left(A\left(x_{1}, \lambda\right), A\left(x_{2}, \lambda\right)+L_{(P, 2)} H\left(B\left(y_{1}, \mu\right), B\left(y_{2}, \mu\right)\right)\right. \\
& +l_{(P, 1)}\left\|\lambda_{1}-\lambda_{2}\right\|_{1}+l_{(P, 2)}\left\|\mu_{1}-\mu_{2}\right\|_{2} \\
\leq & L_{(P, 1)} L_{A}\left\|x_{1}-x_{2}\right\|_{1}+L_{(P, 2)} L_{B}\left\|y_{1}-y_{2}\right\|_{2}+l_{(P, 1)}\left\|\lambda_{1}-\lambda_{2}\right\|_{1} \\
& +l_{(P, 2)}\left\|\mu_{1}-\mu_{2}\right\|_{2},
\end{aligned}
$$

and

$$
\begin{aligned}
& \| R_{1} \circ g_{1}\left(x_{1}, \lambda\right)-R_{1} \circ g_{1}\left(x_{2}, \lambda\right)-\rho_{1}\left(F\left(x_{1}, y_{1}, \lambda, \mu\right)-F\left(x_{2}, y_{2}, \lambda, \mu\right)\right. \\
& \left.-P\left(u_{1}, v_{1}, \lambda, \mu\right)+P\left(u_{2}, v_{2}, \lambda, \mu\right)\right) \|_{1} \\
\leq & \left\|R_{1} \circ g_{1}\left(x_{1}, \lambda\right)-R_{1} \circ g_{1}\left(x_{2}, \lambda\right)-\rho_{1}\left(F\left(x_{1}, y_{1}, \lambda, \mu\right)-F\left(x_{2}, y_{1}, \lambda, \mu\right)\right)\right\|_{1} \\
& +\rho_{1}\left\|F\left(x_{2}, y_{1}, \lambda, \mu\right)-F\left(x_{2}, y_{2}, \lambda, \mu\right)\right\|_{1} \\
& \left.+\rho_{1} \| P\left(u_{1}, v_{1}, \lambda, \mu\right)-P\left(u_{2}, v_{2}, \lambda, \mu\right)\right) \|_{1} .
\end{aligned}
$$

Further, since $F$ is $\xi_{1}$-strongly accretive with respect to $R_{1} \circ g_{1}$ in the first argument and $\left(L_{(F, 1)}, L_{(F, 2)}, l_{(F, 1)}, l_{(F, 2)}\right)$-mixed Lipschitz continuous and $R_{1} \circ g_{1}$ is $\left(L_{R_{1} \circ g_{1}}, l_{R_{1} \circ g_{1}}\right)$-mixed Lipschitz continuous, then

$$
\begin{gathered}
\left\|R_{1} \circ g_{1}\left(x_{1}, \lambda\right)-R_{1} \circ g_{1}\left(x_{2}, \lambda\right)-\rho_{1}\left(F\left(x_{1}, y_{1}, \lambda, \mu\right)-F\left(x_{2}, y_{1}, \lambda, \mu\right)\right)\right\|_{1}^{2} \\
\leq\left\|R_{1} \circ g_{1}\left(x_{1}, \lambda\right)-R_{1} \circ g_{1}\left(x_{2}, \lambda\right)\right\|^{2}-2 \rho_{1}\left\langle F\left(x_{1}, y_{1}, \lambda, \mu\right)-F\left(x_{2}, y_{1}, \lambda, \mu\right),\right. \\
J_{1}\left(R_{1} \circ g_{1}\left(x_{1}, \lambda\right)-R_{1} \circ g_{1}\left(x_{2}, \lambda\right)-\rho_{1}\left(F\left(x_{1}, y_{1}, \lambda, \mu\right)-F\left(x_{2}, y_{1}, \lambda, \mu\right)\right)\right\rangle_{1} \\
\leq L_{R_{1} \circ g_{1}}^{2}\left\|x_{1}-x_{2}\right\|^{2}-2 \rho_{1}\left\langle F\left(x_{1}, y_{1}, \lambda, \mu\right)-F\left(x_{2}, y_{1}, \lambda, \mu\right),\right. \\
\left.\quad J_{1}\left(R_{1} \circ g_{1}\left(x_{1}, \lambda\right)-R_{1} \circ g_{1}\left(x_{2}, \lambda\right)\right)\right\rangle_{1} \\
-2 \rho_{1}\left\langle F\left(x_{1}, y_{1}, \lambda, \mu\right)-F\left(x_{2}, y_{1}, \lambda, \mu\right), J_{1}\left(R_{1} \circ g_{1}\left(x_{1}, \lambda\right)-R_{1} \circ g_{1}\left(x_{2}, \lambda\right)\right)\right.
\end{gathered}
$$




$$
\begin{aligned}
& \left.-\rho_{1}\left(F\left(x_{1}, y_{1}, \lambda, \mu\right)-F\left(x_{2}, y_{1}, \lambda, \mu\right)\right)-J_{1}\left(R_{1} \circ g_{1}\left(x_{1}, \lambda\right)-R_{1} \circ g_{1}\left(x_{2}, \lambda\right)\right)\right\rangle_{1} \\
\leq & L_{R_{1} \circ g_{1}}^{2}\left\|x_{1}-x_{2}\right\|^{2}-2 \rho_{1} \xi_{1}\left\|x_{1}-x_{2}\right\|_{1}^{2} \\
& +64 c_{1} \rho_{1}^{2}\left\|F\left(x_{1}, y_{1}, \lambda, \mu\right)-F\left(x_{2}, y_{1}, \lambda, \mu\right)\right\|_{1}^{2} \\
\leq & L_{R_{1} \circ g_{1}}^{2}\left\|x_{1}-x_{2}\right\|^{2}-2 \rho_{1} \xi_{1}\left\|x_{1}-x_{2}\right\|_{1}^{2}+64 c_{1} \rho_{1}^{2} L_{(F, 1)}^{2}\left\|x_{1}-x_{2}\right\|_{1}^{2} \\
\leq & \left(L_{R_{1} \circ g_{1}}^{2}-2 \rho_{1} \xi_{1}+64 c_{1} \rho_{1}^{2} L_{(F, 1)}^{2}\right)\left\|x_{1}-x_{2}\right\|_{1}^{2} .
\end{aligned}
$$

Since, $F$ is $\left(L_{(F, 1)}, L_{(F, 2)}, l_{(F, 1)}, l_{(F, 2)}\right)$-mixed Lipschitz continuous, then we have

$$
\left\|F\left(x_{2}, y_{1}, \lambda, \mu\right)-F\left(x_{2}, y_{2}, \lambda, \mu\right)\right\|_{1} \leq L_{(F, 2)}\left\|y_{1}-y_{2}\right\|_{2} .
$$

It follows from (4.9)-(4.12) and (4.14), we have

$$
\begin{aligned}
& \left\|t_{1}-t_{2}\right\|_{1} \\
\leq & \sqrt{1-2 s_{1}+64 c_{1} L_{g_{1}}^{2}}\left\|x_{1}-x_{2}\right\|_{1} \\
& +\frac{1}{\gamma_{1}} \sqrt{L_{R_{1} \circ g_{1}}^{2}-2 \rho_{1} \xi_{1}+64 c_{1} \rho_{1}^{2} L_{(F, 1)}^{2}}\left\|x_{1}-x_{2}\right\|_{1} \\
& +\frac{\rho_{1}}{\gamma_{1}} L_{(F, 2)}\left\|y_{1}-y_{2}\right\|_{2}+\frac{\rho_{1}}{\gamma_{1}}\left[L_{(P, 1)} L_{A}\left\|x_{1}-x_{2}\right\|_{1}+L_{(P, 2)} L_{B}\left\|y_{1}-y_{2}\right\|_{2}\right] \\
\leq & \left(\sqrt{1-2 s_{1}+64 c_{1} L_{g_{1}}^{2}}+\frac{\rho_{1}}{\gamma_{1}} \mathrm{~L}_{(P, 1)} L_{A}+\frac{1}{\gamma_{1}} \sqrt{L_{R_{1} \circ g_{1}}^{2}-2 \rho_{1} \xi_{1}+64 c_{1} \rho_{1}^{2} L_{(F, 1)}^{2}}\right) \\
& \times\left\|x_{1}-x_{2}\right\|_{1}+\frac{\rho_{1}}{\gamma_{1}}\left(L_{(F, 2)}+L_{(P, 2)} L_{B}\right)\left\|y_{1}-y_{2}\right\|_{2} .
\end{aligned}
$$

From (4.5) and (4.8) and Lemma 2.3, we have

$$
\begin{aligned}
& \left\|p_{1}-p_{2}\right\|_{2} \\
\leq & \left\|y_{1}-y_{2}-\left(g_{2}\left(y_{1}, \mu\right)-g_{2}\left(y_{2}, \mu\right)\right)\right\|_{2} \\
& +\| J_{\rho_{2}}^{N(\cdot, \mu)}\left[R_{2} \circ g_{2}\left(y_{1}, \mu\right)-\rho_{2} G\left(x_{1}, y_{1}, \lambda, \mu\right)+\rho_{2} Q\left(w_{1}, z_{1}, \lambda, \mu\right)+\rho_{2} f_{2}\right] \\
& -J_{\rho_{2}}^{N(\cdot, \mu)}\left[R_{2} \circ g_{2}\left(y_{2}, \mu\right)-\rho_{2} G\left(x_{2}, y_{2}, \lambda, \mu\right)+\rho_{2} Q\left(w_{2}, z_{2}, \lambda, \mu\right)+\rho_{2} f_{2}\right] \|_{2} \\
\leq & \left\|y_{1}-y_{2}-\left(g_{2}\left(y_{1}, \mu\right)-g_{2}\left(y_{2}, \mu\right)\right)\right\|_{2}+\frac{1}{\gamma_{2}} \| R_{2} \circ g_{2}\left(y_{1}, \mu\right)-R_{2} \circ g_{2}\left(y_{2}, \mu\right) \\
& -\rho_{2}\left(G\left(x_{1}, y_{1}, \lambda, \mu\right)-G\left(x_{2}, y_{2}, \lambda, \mu\right)-Q\left(w_{1}, z_{1}, \lambda, \mu\right)+Q\left(w_{2}, z_{2}, \lambda, \mu\right) \|_{2} .\right.
\end{aligned}
$$

Since $g_{2}$ is $s_{2}$-strongly accretive and $\left(L_{g_{2}}, l_{g_{2}}\right)$-mixed Lipschitz continuous, we have $Q$ is $\left(L_{(Q, 1)}, L_{(Q, 2)}, l_{(Q, 1)}, l_{(Q, 2)}\right)$-mixed Lipschitz continuous and $H$ Lipschitz continuity of set-valued mappings $C, D$, we have

$$
\begin{aligned}
& \left\|Q\left(w_{1}, z_{1}, \lambda_{1}, \mu_{1}\right)-Q\left(w_{2}, z_{2}, \lambda_{2}, \mu_{2}\right)\right\|_{1} \\
\leq & L_{(Q, 1)}\left\|w_{1}-w_{2}\right\|_{1}+L_{(Q, 2)}\left\|z_{1}-z_{2}\right\|_{2}+l_{(Q, 1)}\left\|\lambda_{1}-\lambda_{2}\right\|_{1}+l_{(Q, 2)}\left\|\mu_{1}-\mu_{2}\right\|_{2}
\end{aligned}
$$




$$
\begin{aligned}
\leq & L_{(Q, 1)} H\left(C\left(x_{1}, \lambda\right), C\left(x_{2}, \lambda\right)+L_{(Q, 2)} H\left(D\left(y_{1}, \mu\right), D\left(y_{2}, \mu\right)\right)\right. \\
& +l_{(Q, 1)}\left\|\lambda_{1}-\lambda_{2}\right\|_{1}+l_{(Q, 2)}\left\|\mu_{1}-\mu_{2}\right\|_{2} \\
\leq & L_{(Q, 1)} L_{C}\left\|x_{1}-x_{2}\right\|_{1}+L_{(Q, 2)} L_{D}\left\|y_{1}-y_{2}\right\|_{2}+l_{(Q, 1)}\left\|\lambda_{1}-\lambda_{2}\right\|_{1} \\
& +l_{(Q, 2)}\left\|\mu_{1}-\mu_{2}\right\|_{2},
\end{aligned}
$$

$$
\begin{aligned}
& \left\|y_{1}-y_{2}-\left(g_{2}\left(y_{1}, \mu\right)-g_{2}\left(y_{2}, \mu\right)\right)\right\|_{2}^{2} \\
\leq & \left\|y_{1}-y_{2}\right\|_{2}^{2}-2\left\langle g_{2}\left(y_{1}, \mu\right)-g_{2}\left(y_{2}, \mu\right), J_{2}\left(y_{1}-y_{2}-\left(g_{2}\left(y_{1}, \mu\right)-g_{2}\left(y_{2}, \mu\right)\right)\right)\right\rangle_{2} \\
\leq & \left\|y_{1}-y_{2}\right\|_{2}^{2}-2\left\langle g_{2}\left(y_{1}, \mu\right)-g_{2}\left(y_{2}, \mu\right), J_{2}\left(y_{1}-y_{2}\right)\right\rangle_{2} \\
& -2\left\langle g_{2}\left(y_{1}, \mu\right)-g_{2}\left(y_{2}, \mu\right), J_{2}\left(y_{1}-y_{2}-\left(g_{2}\left(y_{1}, \mu\right)-g_{2}\left(y_{2}, \mu\right)\right)\right)-J_{2}\left(y_{1}-y_{2}\right)\right\rangle_{2} \\
\leq & \left\|y_{1}-y_{2}\right\|_{2}^{2}-2 s_{2}\left\|y_{1}-y_{2}\right\|^{2}+64 c_{2} L_{g_{2}}^{2}\left\|y_{1}-y_{2}\right\|_{2}^{2} \\
\leq & \left(1-2 s_{2}+64 c_{2} L_{g_{2}}^{2}\right)\left\|y_{1}-y_{2}\right\|_{2}^{2} .
\end{aligned}
$$

Now,

$$
\begin{aligned}
& \| R_{2} \circ g_{2}\left(y_{1}, \mu\right)-R_{2} \circ g_{2}\left(y_{2}, \mu\right)-\rho_{2}\left(G\left(x_{1}, y_{1}, \lambda, \mu\right)-G\left(x_{2}, y_{2}, \lambda, \mu\right)\right. \\
& \left.-Q\left(w_{1}, z_{1}, \lambda, \mu\right)+Q\left(w_{2}, z_{2}, \lambda, \mu\right)\right) \|_{2} \\
\leq & \left\|R_{2} \circ g_{2}\left(y_{1}, \mu\right)-R_{2} \circ g_{2}\left(y_{2}, \mu\right)-\rho_{2}\left(G\left(x_{1}, y_{1}, \lambda, \mu\right)-G\left(x_{1}, y_{2}, \lambda, \mu\right)\right)\right\|_{2} \\
& +\rho_{2}\left\|G\left(x_{1}, y_{2}, \lambda, \mu\right)-G\left(x_{2}, y_{2}, \lambda, \mu\right)\right\|_{2} \\
& \left.+\rho_{2} \| Q\left(w_{1}, z_{1}, \lambda, \mu\right)-Q\left(w_{2}, z_{2}, \lambda, \mu\right)\right) \|_{2} .
\end{aligned}
$$

Further, since $G$ is $\xi_{2}$-strongly accretive with respect to $R_{2} \circ g_{2}$ in the second argument and $\left(L_{(G, 1)}, L_{(G, 2)}, l_{(G, 1)}, l_{(G, 2)}\right)$-mixed Lipschitz continuous and $R_{2} \circ g_{2}$ is $\left(L_{R_{2} \circ g_{2}}, l_{R_{2} \circ g_{2}}\right)$-mixed Lipschitz continuous, then we have

$$
\begin{aligned}
& \left\|R_{2} \circ g_{2}\left(y_{1}, \mu\right)-R_{2} \circ g_{2}\left(y_{2}, \mu\right)-\rho_{2}\left(G\left(x_{1}, y_{1}, \lambda, \mu\right)-G\left(x_{1}, y_{2}, \lambda, \mu\right)\right)\right\|_{2}^{2} \\
\leq & \left\|R_{2} \circ g_{2}\left(y_{1}, \mu\right)-R_{2} \circ g_{2}\left(y_{2}, \mu\right)\right\|_{2}^{2}-2 \rho_{2}\left\langle G\left(x_{1}, y_{1}, \lambda, \mu\right)-G\left(x_{1}, y_{2}, \lambda, \mu\right),\right. \\
& \left.J_{2}\left(R_{2} \circ g_{2}\left(y_{1}, \mu\right)-R_{2} \circ g_{2}\left(y_{2}, \mu\right)-\rho\left(G\left(x_{1}, y_{1}, \lambda, \mu\right)-G\left(x_{1}, y_{2}, \lambda, \mu\right)\right)\right)\right\rangle_{2} \\
\leq & L_{R_{2} \circ g_{2}}^{2}\left\|y_{1}-y_{2}\right\|_{2}^{2}-2 \rho_{2}\left\langle G\left(x_{1}, y_{1}, \lambda, \mu\right)-G\left(x_{1}, y_{2}, \lambda, \mu\right), J_{2}\left(R_{2} \circ g_{2}\left(y_{1}, \mu\right)\right.\right. \\
& \left.\left.-R_{2} \circ g_{2}\left(y_{2}, \mu\right)\right)\right\rangle_{2}-2 \rho_{2}\left\langle G\left(x_{1}, y_{1}, \lambda, \mu\right)-G\left(x_{1}, y_{2}, \lambda, \mu\right), J_{2}\left(R_{2} \circ g_{2}\left(y_{1}, \mu\right)\right.\right. \\
& \left.-R_{2} \circ g_{2}\left(y_{2}, \mu\right)-\rho_{2}\left(G\left(x_{1}, y_{1}, \lambda, \mu\right)-G\left(x_{1}, y_{2}, \lambda, \mu\right)\right)\right)-J_{2}\left(R_{2} \circ g_{2}\left(y_{1}, \mu\right)\right. \\
& \left.\left.-R_{2} \circ g_{2}\left(y_{2}, \mu\right)\right)\right\rangle_{2} \\
\leq & L_{R_{2} \circ g_{2}}^{2}\left\|y_{1}-y_{2}\right\|^{2}-2 \rho_{2} \xi_{2}\left\|y_{1}-y_{2}\right\|_{1}^{2} \\
& +64 c_{2} \rho_{2}^{2}\left\|G\left(x_{1}, y_{1}, \lambda, \mu\right)-G\left(x_{1}, y_{2}, \lambda, \mu\right)\right\|_{2}^{2} \\
\leq & L_{R_{2} \circ g_{2}}^{2}\left\|y_{1}-y_{2}\right\|^{2}-2 \rho_{2} \xi_{2}\left\|y_{1}-y_{2}\right\|_{1}^{2}+64 c_{2} \rho_{2}^{2} L_{(G, 1)}^{2}\left\|y_{1}-y_{2}\right\|_{2}^{2} \\
\leq & \left(L_{R_{2} \circ g_{2}}^{2}-2 \rho_{2} \xi_{2}+64 c_{2} \rho_{2}^{2} L_{(G, 1)}^{2}\right)\left\|y_{1}-y_{2}\right\|_{2}^{2} .
\end{aligned}
$$


Since, $G$ is $\left(L_{(G, 1)}, L_{(G, 2)}, l_{(G, 1)}, l_{(G, 2)}\right)$-mixed Lipschitz continuous, then we have

$$
\left\|G\left(x_{1}, y_{2}, \lambda, \mu\right)-G\left(x_{2}, y_{2}, \lambda, \mu\right)\right\|_{2} \leq L_{(G, 1)}\left\|x_{1}-x_{2}\right\|_{1} .
$$

It follows from (4.12), (4.16)-(4.21), we have

$$
\begin{aligned}
& \left\|p_{1}-p_{2}\right\|_{2} \\
\leq & \sqrt{1-2 s_{2}+64 c_{2} L_{g_{2}}^{2}}\left\|y_{1}-y_{2}\right\|_{2} \\
& +\frac{1}{\gamma_{2}} \sqrt{L_{R_{2} \circ g_{2}}^{2}-2 \rho_{2} \xi_{2}+64 c_{2} \rho_{2}^{2} L_{(G, 2)}^{2}}\left\|y_{1}-y_{2}\right\|_{2} \\
& +\frac{\rho_{2}}{\gamma_{2}} L_{(G, 1)}\left\|x_{1}-x_{2}\right\|_{1}+\frac{\rho_{2}}{\gamma_{2}}\left[L_{(Q, 1)} L_{C}\left\|x_{1}-x_{2}\right\|_{1}+L_{(Q, 2)} L_{D}\left\|y_{1}-y_{2}\right\|_{2}\right] \\
\leq & \left(\sqrt{1-2 s_{2}+64 c_{2} L_{g_{2}}^{2}}+\frac{\rho_{2}}{\gamma_{2}} L_{(Q, 2)} L_{D}+\frac{1}{\gamma_{2}} \sqrt{L_{R_{2} \circ g_{2}}^{2}-2 \rho_{2} \xi_{2}+64 c_{2} \rho_{2}^{2} L_{(G, 2)}^{2}}\right) \\
& \times\left\|y_{1}-y_{2}\right\|_{2}+\frac{\rho_{2}}{\gamma_{2}}\left(L_{(G, 1)}+L_{(Q, 1)} L_{C}\right)\left\|x_{1}-x_{2}\right\|_{1} .
\end{aligned}
$$

From (4.15) and (4.22), we have

$$
\begin{aligned}
\left\|\left(t_{1}, p_{1}\right)-\left(t_{2}, p_{2}\right)\right\|_{*} & =\left\|t_{1}-t_{2}\right\|_{1}+\left\|p_{1}-p_{2}\right\|_{2} \\
& \leq k_{1}\left\|x_{1}-x_{2}\right\|_{1}+k_{2}\left\|y_{1}-y_{2}\right\|_{2} \\
& \leq \max \left\{k_{1}, k_{2}\right\}\left(\left\|x_{1}-x_{2}\right\|_{1}+\left\|y_{1}-y_{2}\right\|_{2}\right),
\end{aligned}
$$

where $E^{*}:=E_{1} \times E_{2}$ is a Banach space with norm $\|\cdot\|_{*}=\|\cdot\|_{1}+\|\cdot\|_{2}$;

$(4.24) k_{1}:=m_{1}+\frac{\rho_{2}}{\gamma_{2}}\left(L_{(G, 1)}+L_{(Q, 1)} L_{C}\right) ; k_{2}:=m_{2}+\frac{\rho_{1}}{\gamma_{1}}\left(L_{(F, 2)}+L_{(P, 2)} L_{B}\right)$;

$m_{1}:=\sqrt{1-2 s_{1}+64 c_{1} L_{g_{1}}^{2}}+\frac{\rho_{1}}{\gamma_{1}} L_{(P, 1)} L_{A}+\frac{1}{\gamma_{1}} \sqrt{L_{R_{1} \circ g_{1}}^{2}-2 \rho_{1} \xi_{1}+64 c_{1} \rho_{1}^{2} L_{(F, 1)}^{2}} ;$

$m_{2}:=\sqrt{1-2 s_{2}+64 c_{2} L_{g_{2}}^{2}}+\frac{\rho_{2}}{\gamma_{2}} L_{(Q, 2)} L_{D}+\frac{1}{\gamma_{2}} \sqrt{L_{R_{2} \circ g_{2}}^{2}-2 \rho_{2} \xi_{2}+64 c_{2} \rho_{2}^{2} L_{(G, 2)}^{2}}$.

Hence, we have

$$
\begin{aligned}
d\left(\left(t_{1}, p_{1}\right), T\left(x_{2}, y_{2}, \lambda, \mu\right)\right) & =\inf _{\left(t_{2}, p_{2}\right) \in T\left(x_{2}, y_{2}, \lambda, \mu\right)}\left\|\left(t_{1}, p_{1}\right)-\left(t_{2}, p_{2}\right)\right\|_{*} \\
& \leq \max \left\{k_{1}, k_{2}\right\}\left(\left\|x_{1}-x_{2}\right\|_{1}+\left\|y_{1}-y_{2}\right\|_{2}\right) .
\end{aligned}
$$

Since $\left(t_{1}, p_{1}\right) \in T\left(x_{1}, y_{1}, \lambda, \mu\right)$ is arbitrary, we obtain

$\sup _{\left(t_{1}, p_{1}\right) \in T\left(x_{1}, y_{1}, \lambda, \mu\right)} d\left(\left(t_{1}, p_{1}\right), T\left(x_{2}, y_{2}, \lambda, \mu\right)\right) \leq \max \left\{k_{1}, k_{2}\right\}\left(\left\|x_{1}-x_{2}\right\|_{1}+\left\|y_{1}-y_{2}\right\|_{2}\right)$. 
By using same argument, we have

$\sup _{\left(t_{2}, p_{2}\right) \in T\left(x_{2}, y_{2}, \lambda, \mu\right)} d\left(\left(t_{2}, p_{2}\right), T\left(x_{1}, y_{1}, \lambda, \mu\right)\right) \leq \max \left\{k_{1}, k_{2}\right\}\left(\left\|x_{1}-x_{2}\right\|_{1}+\left\|y_{1}-y_{2}\right\|_{2}\right)$.

By definition of the Hausdorff metric $H$ on $C\left(E_{1} \times E_{2}\right)$, we obtain that for all $\left(x_{i}, y_{i}, \lambda, \mu\right) \in E_{1} \times E_{2} \times \Omega_{1} \times \Omega_{2}$,

$$
H\left(T\left(x_{1}, y_{1}, \lambda, \mu\right), T\left(x_{2}, y_{2}, \lambda, \mu\right)\right) \leq \max \left\{k_{1}, k_{2}\right\}\left(\left\|\left(x_{1}, y_{1}\right)-\left(x_{2}, y_{2}\right)\right\|_{*}\right)
$$

that is, $T(x, y, \lambda, \mu)$ is a uniform $\theta-H$-contraction mapping with respect to $(\lambda, \mu) \in \Omega_{1} \times \Omega_{2}$, where $\theta=\max \left\{k_{1}, k_{2}\right\}$. Also, it follows from condition (4.24) that $\theta<1$ and hence $T(x, y, \lambda, \mu)$ is a set-valued contraction mapping which is uniform with respect to $(\lambda, \mu) \in \Omega_{1} \times \Omega_{2}$. By a fixed point theorem of Nadler [29], for each $(\lambda, \mu) \in \Omega_{1} \times \Omega_{2}, T(x, y, \lambda, \mu)$ has a fixed point $(x, y) \in$ $E_{1} \times E_{2}$, and hence Lemma 3.1 ensures that $S(\lambda, \mu) \neq \emptyset$. Further, for any sequence $\left\{x_{n}, y_{n}\right\} \subseteq S(\lambda, \mu)$ with $\lim _{n \rightarrow \infty}\left(x_{n}, y_{n}\right)=\left(x_{0}, y_{0}\right)$, we have $\left(x_{n}, y_{n}\right) \in$ $T\left(x_{n}, y_{n}, \lambda, \mu\right)$ for all $n \geq 1$. By virtue of (4.30), we have that

$$
\begin{aligned}
& d\left(\left(x_{0}, y_{0}\right), T\left(x_{0}, y_{0}, \lambda, \mu\right)\right) \\
\leq & \left\|\left(x_{0}, y_{0}\right)-\left(x_{n}, y_{n}\right)\right\|_{*}+H\left(T\left(x_{n}, y_{n}, \lambda, \mu\right), T\left(x_{0}, y_{0}, \lambda, \mu\right)\right) \\
\leq & (1+\theta)\left\|\left(x_{n}, y_{n}\right)-\left(x_{0}, y_{0}\right)\right\|_{*} \rightarrow 0 \text { as } n \rightarrow \infty,
\end{aligned}
$$

that is, $\left(x_{0}, y_{0}\right) \in T\left(x_{0}, y_{0}, \lambda, \mu\right)$ and hence $\left(x_{0}, y_{0}\right) \in S(\lambda, \mu)$. Thus $S(\lambda, \mu)$ is closed in $E_{1} \times E_{2}$. This completes the proof.

Theorem 4.2. For each $i=1,2$, let $E_{i}$ be real uniformly smooth Banach space with $\rho_{E_{i}}(t) \leq c_{i} t^{2}$ for some $c_{i}>0$; let the mappings $g_{i}, R_{i} \circ g_{i}, A, B, C, D, F$, $G, P, Q$ be same as in Theorem 4.1 and condition (4.1) holds and there exist constants $\delta_{1}, \delta_{2}>0$ such that

$$
\begin{aligned}
& \left.\left\|J_{\rho_{1}}^{M(\cdot, \lambda)}(x)-J_{\rho_{1}(\cdot, \bar{\lambda})}^{M(x) \|_{1}} \leq \delta_{1}\right\| \lambda-\bar{\lambda} \|_{1} \text { for all } x \in E_{1}, \quad \lambda, \bar{\lambda} \in \Omega_{1}, \quad\right\} \text {. } \\
& \left.\left\|J_{\rho_{2}}^{N(\cdot, \mu)}(y)-J_{\rho_{2}}^{M(\cdot, \bar{\mu})}(y)\right\|_{2} \leq \delta_{2}\|\mu-\bar{\mu}\|_{2} \text { for all } y \in E_{2}, \mu, \bar{\mu} \in \Omega_{2}, \quad\right\}
\end{aligned}
$$

Then the solution set $S(\lambda, \mu)$ of SPGQVLI (3.1) is a H-Lipschitz continuous mapping for $\Omega_{1} \times \Omega_{2}$ into $E_{1} \times E_{2}$.

Proof. For each $(\lambda, \mu),(\bar{\lambda}, \bar{\mu}) \in \Omega_{1} \times \Omega_{2}$, it follows from Theorem $4.1, S(\lambda, \mu)$ and $S(\bar{\lambda}, \bar{\mu})$ are both non-empty and closed subsets of $E_{1} \times E_{2}$. Again by Theorem 4.1, $T(x, y, \lambda, \mu)$ and $T(x, y, \bar{\lambda}, \bar{\mu})$ are both set-valued $\theta-H$-contraction mappings with same contractive constant $\theta \in(0,1)$. By Lemma 2.4, we obtain (4.33)

$$
H(S(\lambda, \mu), S(\bar{\lambda}, \bar{\mu})) \leq\left(\frac{1}{1-\theta}\right) \sup _{(x, y) \in E_{1} \times E_{2}} H(T(x, y, \lambda, \mu), T(x, y, \bar{\lambda}, \bar{\mu})
$$

where $\theta$ is given by (4.1). 
Now, for any $\left(i_{1}, l_{1}\right) \in T(x, y, \lambda, \mu)$, there exist $u=u(x, \lambda) \in A(x, \lambda), v=$ $v(y, \mu) \in B(y, \mu), w=w(x, \lambda) \in C(x, \lambda), z=z(y, \mu) \in D(y, \mu)$ satisfying $(4.34)$

$i_{1}=x-g_{1}(x, \lambda)+J_{\rho_{1}}^{M(\cdot, \lambda)}\left[R_{1} \circ g_{1}(x, \lambda)-\rho_{1} F(x, y, \lambda, \mu)+\rho_{1} P(u, v, \lambda, \mu)+\rho_{1} f_{1}\right]$, and

$l_{1}=y-g_{2}(y, \mu)+J_{\rho_{2}}^{N(\cdot, \mu)}\left[R_{2} \circ g_{2}(y, \mu)-\rho_{2} G(x, y, \lambda, \mu)+\rho_{2} Q(w, z, \lambda, \mu)+\rho_{2} f_{2}\right]$.

It follows from the compactness of $A\left(x_{2}, \lambda\right), B\left(y_{2}, \mu\right), C\left(x_{2}, \lambda\right)$ and $D\left(y_{2}, \mu\right)$ and $H$-Lipschitz continuity of $A, B, C, D$ that there exist $\bar{u}=u(x, \bar{\lambda}) \in A(x, \bar{\lambda})$, $\bar{v}=v(y, \bar{\mu}) \in B(y, \bar{\mu}), \bar{w}=w(x, \bar{\lambda}) \in C(x, \bar{\lambda}), \bar{z}=z(y, \bar{\mu}) \in D(y, \bar{\mu})$ such that

$$
\begin{aligned}
&\|u-\bar{u}\|_{1} \leq H(A(x, \lambda), A(x, \bar{\lambda})) \leq l_{A}\|\lambda-\bar{\lambda}\|, \\
&\|v-\bar{v}\|_{2} \leq H(B(y, \mu), B(y, \bar{\mu})) \leq l_{B}\|\mu-\bar{\mu}\|, \\
&\|w-\bar{w}\|_{1} \leq H(C(x, \lambda), C(x, \bar{\lambda})) \leq l_{C}\|\lambda-\bar{\lambda}\|, \\
&\|z-\bar{z}\|_{2} \leq H(D(y, \mu), D(y, \bar{\mu})) \leq l_{D}\|\mu-\bar{\mu}\| .
\end{aligned}
$$

Let

$i_{2}=x-g_{1}(x, \bar{\lambda})+J_{\rho_{1}}^{M(\cdot, \bar{\lambda})}\left[R_{1} \circ g_{1}(x, \bar{\lambda})-\rho_{1} F(x, y, \bar{\lambda}, \bar{\mu})+\rho_{1} P(\bar{u}, \bar{v}, \bar{\lambda}, \bar{\mu})+\rho_{1} f_{1}\right]$, and

$l_{2}=y-g_{2}(y, \bar{\mu})+J_{\rho_{2}}^{N(\cdot, \bar{\mu})}\left[R_{2} \circ g_{2}(y, \bar{\mu})-\rho_{2} G(x, y, \bar{\lambda}, \bar{\mu})-\rho_{2} Q(\bar{w}, \bar{z}, \bar{\lambda}, \bar{\mu})+\rho_{2} f_{2}\right]$.

Clearly, $\left(i_{2}, l_{2}\right) \in T(x, y, \bar{\lambda}, \bar{\mu})$.

From (4.32), (4.34) and (4.37) and Lemma 2.3, we have

$$
\begin{aligned}
& \left\|i_{1}-i_{2}\right\|_{1} \\
\leq & \left\|g_{1}(x, \lambda)-g_{1}(x, \bar{\lambda})\right\| \\
& +\| J_{\rho_{1}}^{M(\cdot, \lambda)}\left[R_{1} \circ g_{1}(x, \lambda)-\rho_{1} F(x, y, \lambda, \mu)+\rho_{1} P(u, v, \lambda, \mu)+\rho_{1} f_{1}\right] \\
& -J_{\rho_{1}}^{M(\cdot, \lambda)}\left[R_{1} \circ g_{1}(x, \bar{\lambda})-\rho_{1} F(x, y, \bar{\lambda}, \bar{\mu})+\rho_{1} P(\bar{u}, \bar{v}, \bar{\lambda}, \bar{\mu})+\rho_{1} f_{1}\right] \|_{1} \\
& +\| J_{\rho_{1}}^{M(\cdot, \lambda)}\left[R_{1} \circ g_{1}(x, \bar{\lambda})-\rho_{1} F(x, y, \bar{\lambda}, \bar{\mu})+\rho_{1} P(\bar{u}, \bar{v}, \bar{\lambda}, \bar{\mu})+\rho_{1} f_{1}\right] \\
& -J_{\rho_{1}}^{M(\cdot, \bar{\lambda})}\left[R_{1} \circ g_{1}(x, \bar{\lambda})-\rho_{1} F(x, y, \bar{\lambda}, \bar{\mu})+\rho_{1} P(\bar{u}, \bar{v}, \bar{\lambda}, \bar{\mu})+\rho_{1} f_{1}\right] \|_{1} \\
\leq & \left\|g_{1}(x, \lambda)-g_{1}(x, \bar{\lambda})\right\| \\
& +\frac{1}{\gamma_{1}} \| R_{1} \circ g_{1}(x, \lambda)-R_{1} \circ g_{1}(x, \bar{\lambda})+\rho_{1}(P(u, v, \lambda, \mu)-P(\bar{u}, \bar{v}, \bar{\lambda}, \bar{\mu})) \\
& -\rho_{1}\left(F(x, y, \lambda, \mu)-F(x, y, \bar{\lambda}, \bar{\mu})\left\|_{1}+\delta_{1}\right\| \lambda-\bar{\lambda} \|_{1}\right. \\
\leq & \left\|g_{1}(x, \lambda)-g_{1}(x, \bar{\lambda})\right\|
\end{aligned}
$$




$$
\begin{aligned}
& +\frac{1}{\gamma_{1}}\left[\left\|R_{1} \circ g_{1}(x, \lambda)-R_{1} \circ g_{1}(x, \bar{\lambda})\right\|_{1}+\rho_{1} \|\left(F(x, y, \lambda, \mu)-F(x, y, \bar{\lambda}, \bar{\mu}) \|_{1}\right.\right. \\
& \left.+\rho_{1}\|P(u, v, \lambda, \mu)-P(\bar{u}, \bar{v}, \bar{\lambda}, \bar{\mu})\|_{1}\right]+\delta_{1}\|\lambda-\bar{\lambda}\|_{1} .
\end{aligned}
$$
have

Since, $F$ is $\left(L_{(F, 1)}, L_{(F, 2)}, l_{(F, 1)}, l_{(F, 2)}\right)$-mixed Lipschitz continuous, then we

$$
\|F(x, y, \bar{\lambda}, \mu)-F(x, y, \bar{\lambda}, \bar{\mu})\|_{1} \leq l_{(F, 1)}\|\mu-\bar{\mu}\|_{2} .
$$

Also, $g_{1}$ is $\left(L_{g_{1}}, l_{g_{1}}\right)$-mixed Lipschitz continuous, then we have

$$
\left\|g_{1}(x, \lambda)-g_{1}(x, \bar{\lambda})\right\|_{1} \leq l_{g_{1}}\|\lambda-\bar{\lambda}\|_{1},
$$

and $R_{1} \circ g_{1}$ is $\left(L_{R_{1} \circ g_{1}}, l_{R \circ g_{1}}\right)$-mixed Lipschitz continuous, then we have

$$
\left\|R_{1} \circ g_{1}(x, \lambda)-R_{2} \circ g_{2}(x, \bar{\lambda})\right\| \leq l_{R_{1} \circ g_{1}}\|\lambda-\bar{\lambda}\|_{1}
$$

From (4.11), (4.39)-(4.41), we have

$$
\begin{aligned}
& \left\|i_{1}-i_{2}\right\|_{1} \\
\leq & l_{g_{1}}\|\lambda-\bar{\lambda}\|_{1}+\frac{1}{\gamma_{1}}\left[l_{R_{1} \circ g_{1}}\|\lambda-\bar{\lambda}\|_{1}+\rho_{1}\left[l_{(F, 1)}\|\lambda-\bar{\lambda}\|_{1}+l_{(F, 2)}\|\mu-\bar{\mu},\|_{2}\right]\right. \\
& +\rho_{1}\left[L_{(P, 1)} l_{A}+l_{(P, 1)}\right]\|\lambda-\bar{\lambda}\|_{1} \\
& \left.+\rho_{1}\left[L_{(P, 2)} l_{B}+l_{(P, 2)}\right]\|\mu-\bar{\mu}\|_{2}\right]+\delta_{1}\|\lambda-\bar{\lambda}\|_{1} \\
\leq & \frac{1}{\gamma_{1}}\left[\gamma_{1} l_{g_{1}}+l_{R_{1} \circ g_{1}}+\rho_{1}\left(l_{(F, 1)}+L_{(P, 1)}+L_{(P, 1)} l_{A}\right)+\delta_{1} \gamma_{1}\right]\|\lambda-\bar{\lambda}\|_{1} \\
& +\frac{\rho_{1}}{\gamma_{1}}\left[l_{(F, 2)}+l_{(P, 2)}+L_{(P, 2)} l_{B}\right]\|\mu-\bar{\mu}\|_{2} .
\end{aligned}
$$

Since, $G$ is $\left(L_{(G, 1)}, L_{(G, 2)}, l_{(G, 1)}, l_{(G, 2)}\right)$-mixed Lipschitz continuous; $g_{2}$ is $\left(L_{g_{2}}, l_{g_{2}}\right)$-mixed Lipschitz continuous; $R_{2} \circ g_{2}$ is $\left(L_{R_{2} \circ g_{2}}, l_{R_{2} \circ g_{2}}\right)$-mixed Lipschitz continuous; and from (4.18), (4.32), (4.35), (4.36), (4.38) and Lemma 2.3, we have

$$
\begin{aligned}
& \left\|l_{1}-l_{2}\right\|_{2} \\
\leq & \left\|g_{2}(y, \mu)-g_{2}(y, \bar{\mu})\right\|_{2} \\
& +\| J_{\rho_{2}}^{N(\cdot, \mu)}\left[R_{2} \circ g_{2}(y, \mu)-\rho_{2} G(x, y, \lambda, \mu)+\rho_{2} Q(w, z, \lambda, \mu)+\rho_{2} f_{2}\right] \\
& -J_{\rho_{2}}^{N(\cdot, \mu)}\left[R_{2} \circ g_{2}(y, \bar{\mu})-\rho_{2} G(x, y, \bar{\lambda}, \bar{\mu})+\rho_{2} Q(\bar{w}, \bar{z}, \bar{\lambda}, \bar{\mu})+\rho_{2} f_{2}\right] \|_{2} \\
& +\| J_{\rho_{2}}^{N(\cdot, \mu)}\left[R_{2} \circ g_{2}(y, \bar{\mu})-\rho_{2} G(x, y, \bar{\lambda}, \bar{\mu})+\rho_{2} Q(\bar{w}, \bar{z}, \bar{\lambda}, \bar{\mu})+\rho_{2} f_{2}\right] \\
& -J_{\rho_{2}}^{N(\cdot, \bar{\mu})}\left[R_{2} \circ g_{2}(y, \bar{\mu})-\rho_{2} G(x, y, \bar{\lambda}, \bar{\mu})+\rho_{2} Q(\bar{w}, \bar{z}, \bar{\lambda}, \bar{\mu})+\rho_{2} f_{2}\right] \|_{2} \\
\leq & l_{g_{2}}\|\mu-\bar{\mu}\|_{2}
\end{aligned}
$$




$$
\begin{aligned}
& +\frac{1}{\gamma_{2}}\left[\left\|R_{2} \circ g_{2}(y, \mu)-R_{2} \circ g_{2}(y, \bar{\mu})\right\|_{2}+\rho_{2}\|G(x,, y, \lambda, \mu)-G(x, y, \bar{\lambda}, \bar{\mu})\|_{2}\right. \\
& \left.+\rho_{2}\|Q(w, z, \lambda, \mu)-Q(\bar{w}, \bar{z}, \bar{\lambda}, \bar{\mu})\|_{2}\right]+\delta_{2}\left\|\mu_{1}-\mu_{2}\right\|_{2} \\
\leq & l_{g_{2}}\|\mu-\bar{\mu}\|_{2}+\frac{1}{\gamma_{2}}\left[l_{R_{2} \circ g_{2}}\|\mu-\bar{\mu}\|_{2}+\rho_{2}\left(l_{(G, 1)}\|\mu-\bar{\mu}\|_{1}+l_{(G, 2)}\|\mu-\bar{\mu},\|_{2}\right)\right. \\
& +\rho_{2}\left[L_{(Q, 1)} l_{C}+\|\lambda-\bar{\lambda}\|_{1}+l_{(Q, 2)} l_{D}\|\mu-\bar{\mu}\|_{2}\right. \\
& \left.+l_{(Q, 1)}\|\lambda-\bar{\lambda}\|_{1}+l_{(Q, 2)}\|\mu-\bar{\mu}\|_{2}\right]+\delta_{2}\left\|\mu_{1}-\overline{\mu_{2}}\right\|_{2} \\
\leq & \frac{1}{\gamma_{2}}\left[\gamma_{2} l_{g_{2}}+l_{R_{2} \circ g_{2}}+\rho_{2}\left(l_{(G, 2)}+l_{(Q, 2)}+l_{(Q, 2)} l_{D}\right)+\delta_{2} \gamma_{2}\right]\left\|\mu_{1}-\bar{\mu}_{2}\right\|_{2} \\
& +\frac{\rho_{2}}{\gamma_{2}}\left[l_{(G, 1)}+l_{(Q, 1)}+L_{(Q, 1)} l_{C}\right]\|\lambda-\bar{\lambda}\|_{1} .
\end{aligned}
$$

From (4.43) and (4.44), we have

$$
\begin{aligned}
\left\|\left(i_{1}, l_{1}\right)-\left(i_{2}, l_{2}\right)\right\|_{*} & =\left\|i_{1}-i_{2}\right\|_{1}+\left\|l_{1}-l_{2}\right\|_{2} \\
& \leq k_{3}\|\lambda-\bar{\lambda}\|_{1}+k_{4}\|\mu-\bar{\mu}\|_{2} \\
& \leq \max \left\{k_{3}, k_{4}\right\}\|(\lambda, \mu)-(\bar{\lambda}, \bar{\mu})\|_{*},
\end{aligned}
$$

where

(4.46) $k_{3}:=m_{3}+l_{(G, 1)}+l_{(Q, 1)}+L_{(Q, 1)} l_{C} ; k_{4}:=m_{4}+l_{(F, 2)}+l_{(P, 2)}+L_{(P, 2)} l_{B}$;

$$
\begin{aligned}
& m_{3}:=\frac{1}{\gamma_{1}}\left[\gamma_{1} l_{g_{1}}+l_{R_{1} \circ g_{1}}+\rho_{1}\left(l_{(F, 1)}+l_{(P, 1)}+L_{(P, 1)} l_{A}\right)+\delta_{1} \gamma_{1}\right] \\
& m_{4}:=\frac{1}{\gamma_{2}}\left[\gamma_{2} l_{g_{2}}+l_{R_{2} \circ g_{2}}+\rho_{2}\left(l_{(G, 2)}+l_{(Q, 2)}+L_{(Q, 2)} l_{D}\right)+\delta_{2} \gamma_{2}\right] .
\end{aligned}
$$

Hence, we have

$$
\begin{aligned}
d\left(\left(i_{1}, l_{1}\right), T(x, y, \bar{\lambda}, \bar{\mu})\right) & =\inf _{\left(i_{2}, l_{2}\right) \in T(x, y, \bar{\lambda}, \bar{\mu})}\left\|\left(i_{1}, l_{1}\right)-\left(i_{2}, l_{2}\right)\right\|_{*} \\
& \leq \max \left\{k_{3}, k_{4}\right\}\|(\lambda, \mu)-(\bar{\lambda}, \bar{\mu})\|_{*} .
\end{aligned}
$$

Since $\left(i_{1}, l_{1}\right) \in T(x, y, \lambda, \mu)$ is arbitrary, we obtain

$$
\sup _{\left(i_{1}, l_{1}\right) \in T(x, y, \lambda, \mu)} d\left(\left(i_{1}, l_{1}\right), T(x, y, \bar{\lambda}, \bar{\mu})\right) \leq \max \left\{k_{3}, k_{4}\right\}\|(\lambda, \mu)-(\bar{\lambda}, \bar{\mu})\|_{*} .
$$

By using same argument, we can prove

$$
\sup _{\left(i_{2}, l_{2}\right) \in T(x, y, \bar{\lambda}, \bar{\mu})} d\left(\left(i_{2}, l_{2}\right), T(x, y, \lambda, \mu)\right) \leq \max \left\{k_{3}, k_{4}\right\}\|(\lambda, \mu)-(\bar{\lambda}, \bar{\mu})\|_{*} .
$$

Hence, it follows that for all $(x, y, \lambda, \mu),(x, y, \bar{\lambda}, \bar{\mu}) \in E_{1} \times E_{2} \times \Omega_{1} \times \Omega_{2}$,

$$
H(T(x, y, \lambda, \mu), T(x, y, \bar{\lambda}, \bar{\mu})) \leq \theta_{1}\|(\lambda, \mu)-(\bar{\lambda}, \bar{\mu})\|_{*},
$$


$\theta_{1}=\max \left\{k_{3}, k_{4}\right\}$ and hence from (4.33) and (4.52) we obtain

$$
H(S(\lambda, \mu), S(\bar{\lambda}, \bar{\mu})) \leq\left(\frac{\theta_{1}}{(1-\theta)}\right)\left(\|(\lambda, \mu)-(\bar{\lambda}, \bar{\mu})\|_{*} .\right.
$$

This implies that $S(\lambda, \mu)$ is $H$-Lipschitz continuous in $(\lambda, \mu) \in \Omega_{1} \times \Omega_{2}$ and completes the proof.

\section{References}

[1] S. Adly, Perturbed algorithms and sensitivity analysis for a general class of variational inclusions, J. Math. Anal. Appl. 201 (1996), no. 2, 609-630.

[2] R. P. Agarwal, Y.-J. Cho, and N.-J. Huang, Sensitivity analysis for strongly nonlinear quasi-variational inclusions, Appl. Math. Lett. 13 (2000), no. 6, 19-24.

[3] R. P. Agarwal, N.-J. Huang, and M.-Y. Tan, Sensitivity analysis for a new system of generalized nonlinear mixed quasi-variational inclusions, Appl. Math. Lett. 17 (2004), no. 3, 345-352.

[4] J. P. Aubin and A. Cellina, Differential Inclusions: Set-valued Maps and Viability Theory, Grundlehren der Mathematischen Wissenschaften [Fundamental Principles of Mathematical Sciences], 264. Springer-Verlag, Berlin, 1984.

[5] C. Baiocchi and A. Capelo, Variational and Quasi-variational Inequalities, John Wiley \& Sons, Inc., New York, 1984.

[6] A. Bensoussan and J. L. Lions, Applications of Variational Inequalities, GauthiersVillars, Bordas, Paris, 1984.

[7] H. Brezis, Équations et inéquations non linéaires dans les espaces vectoriels en dualité, Ann. Inst. Fourier (Grenoble) 18 (1968), fasc. 1, 115-175.

[8] C. E. Chidume, K. R. Kazmi, and H. Zegeye, Iterative approximation of a solution of a general variational-like inclusion in Banach spaces, Int. J. Math. Math. Sci. 2004 (2004), no. 21-24, 1159-1168.

[9] I. Cioreneseu, Geometry of Banach Spaces, Duality Mappings and Nonlinear Problems, Kluwer Academic Publishers Group, Dordrecht, 1990.

[10] J. Crank, Free and Moving Boundary Problems, The Clarendon Press, Oxford University Press, New York, 1984.

[11] S. Dafermos, Sensitivity analysis in variational inequalities, Math. Oper. Res. 13 (1988), no. $3,421-434$.

[12] K. Demling, Nonlinear Functional Analysis, Springer-Verlag, Berlin, 1985.

[13] V. F. Demyanov, G. E. Stovroulakis, L. N. Poyakova, and P. D. panogiotopoulos, Quasidifferentiability and Nonsmooth Modelling in Mechanics, Engineering and Economics, Nonconvex Optimization and its Applications, 10. Kluwer Academic Publishers, Dordrecht, 1996.

[14] X.-P. Ding, Sensitivity analysis for generalized nonlinear implicit quasi-variational inclusions, Appl. Math. Lett. 17 (2004), no. 2, 225-235.

[15] X.-P. Ding and C. L. Luo, On parametric generalized quasi-variational inequalities, J. Optim. Theory Appl. 100 (1999), no. 1, 195-205.

[16] Y.-P. Fang and N.-J. Huang, H-accretive operators and resolvent operator technique for solving variational inclusions in Banach spaces, Appl. Math. Lett. 17 (2004), no. 6, 647-653.

[17] G. Fichera, Problemi elastostatici con vincoli unilaterali: Il problema di Signorini con ambigue condizioni al contorno, Atti Accad. Naz. Lincei Mem. Cl. Sci. Fis. Mat. Natur. Sez. I (8) 7 (1963/1964), 91-140.

[18] F. Giannessi and A. Maugeri, Variational inequalities and network equilibrium problems, Proceedings of the conference held in Erice, June 19-25, 1994, Plenum Press, New York, 1995. 
[19] R. Glowinski, Numerical Methods For Nonlinear Variational Problems, Springer-Verlag, New York, 1984.

[20] R. Glowinski, J. L. Lions, and R. Tremolieres, Numerical Analysis of Variational Inequalities, North-Holland Publishing Co., Amsterdam-New York, 1981.

[21] K. Goebel and S. Reich, Uniform Convexity, Hyperbolic Geometry and Nonexpansive Mappings, Marcel Dekker, Inc., New York, 1984.

[22] K. R. Kazmi and F. A. Khan, Iterative approximation of a unique solution of a system of variational-like inclusions in real q-uniformly smooth Banach spaces, Nonlinear Anal. 67 (2007), no. 3, 917-929.

[23] _ Sensitivity analysis for parametric generalized implicit quasi-variational-like inclusions involving P- $\eta$-accretive mappings, J. Math. Anal. Appl. 337 (2008), no. 2, $1198-1210$.

[24] _ Sensitivity analysis for parametric general set-valued mixed variational-like inequality in uniformly smooth Banach space, Math. Inequal. Appl. 10 (2007), no. 2, 403-415.

[25] T. C. Lim, On fixed point stability for set-valued contractive mappings with applications to generalized differential equations, J. Math. Anal. Appl. 110 (1985), no. 2, 436-441.

[26] Z. Liu, L. Debnath, S. M. Kang, and J. S. Ume, Sensitivity analysis for parametric completely generalized nonlinear implicit quasivariational inclusions, J. Math. Anal. Appl. 277 (2003), no. 1, 142-154.

[27] J. J. Moreau, Proximiteet dualite dans un espace hilbertien, Bull. Soc. Math. France 93 (1965), 273-299.

[28] R. N. Mukherjee and H. L. Verma, Sensitivity analysis of generalized variational inequalities, J. Math. Anal. Appl. 167 (1992), no. 2, 299-304.

[29] S. B. Nadler, Multi-valued contraction mappings, Pacific J. Math. 30 (1969), 475-488.

[30] M. A. Noor and K. I. Noor, Sensitivity analysis for quasi-variational inclusions, J. Math. Anal. Appl. 236 (1999), no. 2, 290-299.

[31] M. A. Noor, Sensitivity analysis framework for general quasi-variational inclusions, Comput. Math. Appl. 44 (2002), no. 8-9, 1175-1181.

[32] J. Y. Park and J. U. Jeong, Parametric generalized mixed variational inequalities, Appl. Math. Lett. 17 (2004), no. 1, 43-48.

[33] J. W. Peng and X. J. Long, Sensitivity analysis for parametric completely generalized strongly nonlinear implicit quasi-variational inclusions, Comput. Math. Appl. 50 (2005), no. 5-6, 869-880.

[34] J. W. Peng and D. Zhu, A new system of generalized mixed quasi-variational inclusions with $(H, \eta)$-monotone operators, J. Math. Anal. Appl. 327 (2007), no. 1, 175-187.

[35] S. M. Robinson, Sensitivity analysis of variational inequalities by normal-map techniques, Variational inequalities and network equilibrium problems (Erice, 1994), 257269, Plenum, New York, 1995.

[36] G. Stampacchia, Formes bilineaires coercitives sur les ensembles convexes, C. R. Acad. Sci. Paris 258 (1964), 4413-4416.

[37] N. D. Yen, Hölder continuity of solutions to a parametric variational inequality, Appl. Math. Optim. 31 (1995), no. 3, 245-255.

Kaleem Raza Kazmi

Department of Mathematics

Aligarh Muslim University

Aligarh 202 002, India

E-mail address: krkazmi@gmail.com 
Faizan Ahmad Khan

Department of Mathematics

Aligarh Muslim University

Aligarh 202 002, India

NAEem Ahmad

Department of Applied Mathematics

Aligarh Muslim University

Aligarh 202 002, India 